\title{
X-Max Large Area SDD Detectors - Creating a real impact on nano-science
}

\author{
C.L. Collins*, J. Holland*, S. R. Burgess*, N. Rowlands** \\ *Oxford Instruments Nanoanalysis, Halifax Road, High Wycombe, HP12 3SE, UK \\ **Oxford Instruments Inc, 300 Baker Avenue, Suite 150, Concord, Massachusetts, USA
}

Energy Dispersive Spectrometry (EDS) has been used for many years to analyse the chemical composition of materials. In recent years, silicon drift (SDD) detectors with significantly improved performance have emerged on the market [1], however, their impact on the analysis of nanomaterials has been limited. Typically, when conventional SDD detectors are used for high count rate analysis the probe size is increased to generate sufficient X-rays. This limits image resolution and makes it difficult to resolve and analyse nano-scale features. Such conditions can also result in sample damage and contamination.

The introduction of X-Max very large area SDDs from Oxford Instruments now promise practical solutions to nano-analysis challenges with SEM. These detectors offer greatly increased solid angle enabling higher count rates to be achieved under low beam current conditions. This is of major significance when low accelerating voltages are used to minimise the X-ray interaction volume to analyse nano-features. Despite the dramatic drop in X-ray yield, large area SDDs can achieve count rates large enough for practical analysis under these conditions enabling even ultralow energy spectra $(2-5 \mathrm{kV})$ to be collected in a fraction of the time required previously. This means mapping can often be used rather than selected point analysis. Data has been collected on a range of nano-structures to investigate the potential applications of very large area SDDs. This study used an $80 \mathrm{~mm}^{2}$ Oxford Instruments X-Max SDD detector.

Figure 1 shows the results of the investigation of boride and carbide nano-inclusions in a nimonic steel. Data was collected for 90 minutes at $5 \mathrm{kV}$. Conventional X-ray maps were constructed for B, $\mathrm{C}$ and Ni using energy windows spanning the FWHM of the peak and combined to create the mix map seen in Figure 1a. A spectrum equivalent to just $0.5 \mathrm{~s}$ of acquisition has been reconstructed from a boride inclusion measuring less than 50nm, showing the capability of the EDS technique to characterise nano-inclusions of this type using very large area SDD (Figure 1b).

The data shown in Figure 2 was collected from an alloy used in the manufacture of glass fibre spinner bowls. Due to the complex nano-structures in this alloy, low accelerating voltage is used to minimise the interaction volume from which X-rays are generated. This limits the EDS lines which can be used for analysis. Figure 2 a shows a $\mathrm{NbL}_{\alpha}$ map acquired using an accelerating voltage of $5 \mathrm{kV}$. With the improved light element performance of the X-Max SDD and the higher count rates that can be generated at low $\mathrm{kV}$, it is possible to create an elemental distribution map using the $\mathrm{NbM}_{\mathrm{z}}$ line at $0.172 \mathrm{keV}$ (Figures $2 \mathrm{~b}$ and $2 \mathrm{c}$ ). Therefore the potential exists to reduce the accelerating voltage to further improve the spatial resolution when investigating these materials.

Figure 3 illustrates how the improved solid angle achieved by X-Max allows mapping of very thin nanolayers in semiconductors used for LEDs and energy generation. The sample is comprised of a series of $\mathrm{Ga}$, In and P-containing layers [2]. Data was collected at $5 \mathrm{kV}$ and $1.6 \mathrm{nA}$ for just 30 minutes. Figure 3 a shows layers as thin as $25 \mathrm{~nm}$ can be mapped using the $\mathrm{InL}_{\alpha}$ line. 
These nano-structures were all investigated using a very large area SDD detector. Significant potential is indicated for all users and in particular, those looking to use X-ray mapping to investigate chemical distribution at the nano-scale. The use of low accelerating voltages and low beam currents is no longer a practical barrier to the use of EDS in these applications.

\section{References}

[1] C.L. Collins et al, Microscopy and Microanalysis, 14 (Suppl. 2), pp 1104-1105

[2] N. Rowlands et al, MSC, Edmonton, June 2007.

(a)

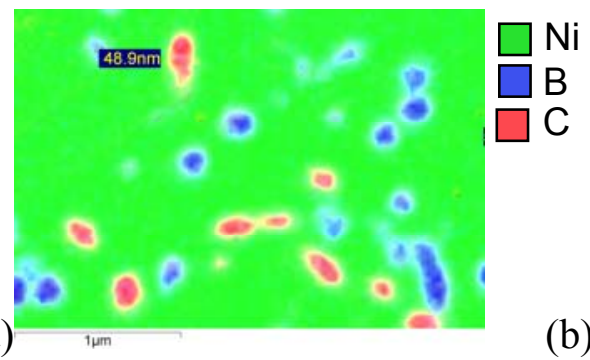

(b)

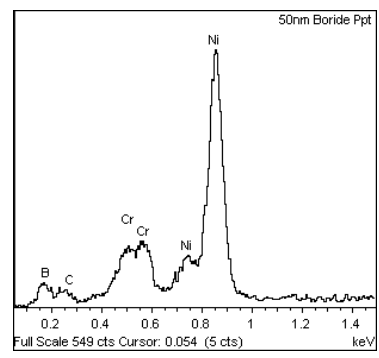

Figure 1. (a) Composite mix map comprised of 3 elemental distribution maps. Blue $=$ Boron, Red $=$ Carbon and Green $=$ Nickel. (b) Reconstructed spectrum from a sub-50nm inclusion showing clear distinction between the boron and carbon peaks.
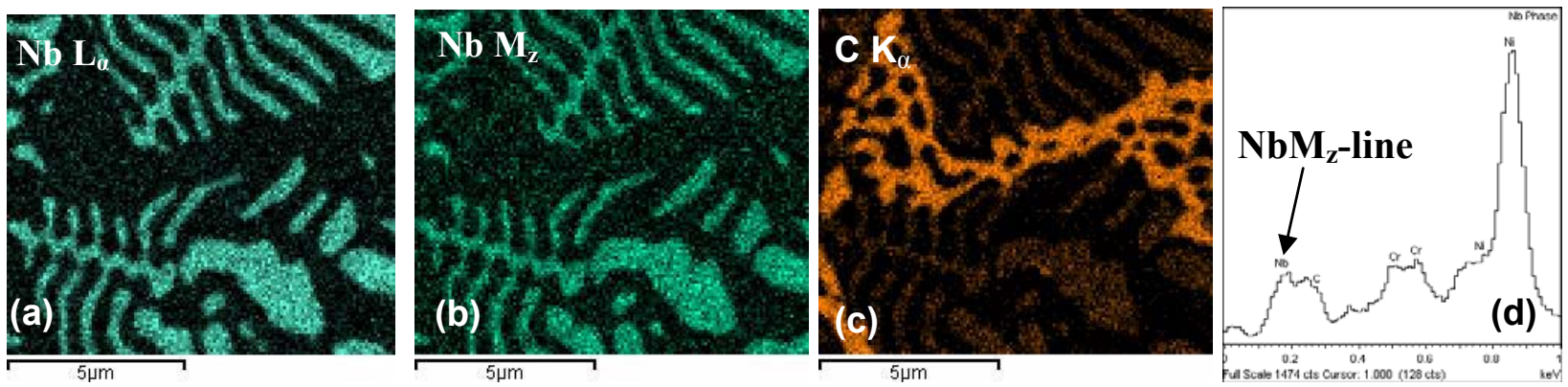

Figure 2. X-ray maps of a spinner bowl sample collected at $5 \mathrm{kV}$. EDS maps constructed using the (a) $\mathrm{NbL}_{\alpha}$-line (b) $\mathrm{NbM}_{\mathrm{z}}$-line (c) $\mathrm{CK}_{\alpha}$-line. (d) Spectrum showing the ultra-low energy $\mathrm{NbM}_{\mathrm{z}}$-line used for the map constructed in Fig. 2b. Note: To remove any potential overlap between the $\mathrm{NbM}_{\mathrm{z}}$ and $\mathrm{CK}_{\alpha}$ maps, map subtraction was used to subtract the $\mathrm{CK}_{\alpha}$ map signal from the $\mathrm{NbM}_{\mathrm{z}}$ map signal.

(a)

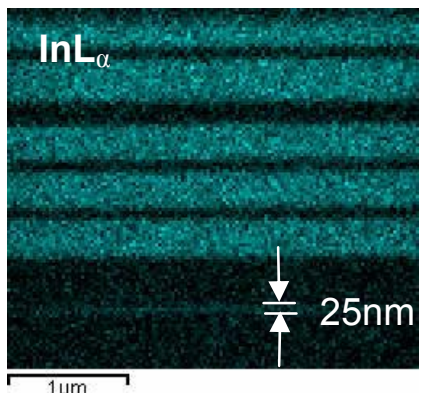

Figure 3. (a) EDS elemental distribution map of a layered semiconductor sample constructed using the $\mathrm{InL}_{\alpha}$-line. 